\title{
Universitários canhotos: dificuldades encontradas e propostas para as atividades cotidianas
}

\author{
Elisângela de Assis Amaro ${ }^{\mathrm{a}}$, Karoline Cipriano Raimundo ${ }^{\mathrm{b}}$, Núbia Tomain Otoni dos Santos ${ }^{\mathrm{b}, \mathrm{c}}$, \\ Patrícia Beatriz Silva Bonfim ${ }^{\mathrm{d}}$, Isabel Aparecida Porcatti de Walsh ${ }^{\mathrm{e}}$, Dernival Bertoncello ${ }^{\mathrm{b}, \mathrm{e}}$ \\ aUniversidade Federal do Triângulo Mineiro - UFTM, Uberaba, MG, Brasil \\ bPrograma de Pós-graduação em Educação Física, Universidade Federal do Triângulo Mineiro - UFTM, \\ Uberaba, MG, Brasil \\ 'Hospital de Clínicas, Universidade Federal do Triângulo Mineiro - UFTM, Uberaba, MG, Brasil \\ dUniversidade de Uberaba - UNIUBE, Uberaba, MG, Brasil \\ eDepartamento de Fisioterapia Aplicada, Universidade Federal do Triângulo Mineiro - UFTM, Uberaba, MG, Brasil
}

\begin{abstract}
Resumo: A presente pesquisa teve como objetivo identificar, por meio de um questionário, as dificuldades cotidianas de universitários canhotos e elencar suas propostas para solucionar os problemas levantados. Participaram deste estudo 135 estudantes canhotos, que responderam um questionário composto por oito perguntas abertas e cinco perguntas fechadas que englobavam informações sobre a lateralidade, atividades estudantis, laborais, de vida diária e a sugestão para melhoria em suas atividades. Verificou-se que as maiores dificuldades relatadas por estudantes universitários canhotos na vida cotidiana, no trabalho e no ambiente estudantil são, respectivamente, abrir latas, má disposição dos objetos no espaço e a inadequação das carteiras escolares. A fim de melhorar a execução de atividades nessas três esferas, as sugestões mais citadas estão relacionadas à melhoria/disponibilidade das carteiras para canhotos e o desenvolvimento de objetos adaptados para esse grupo. O estudo indica dados sobre dificuldades e sugestões que são coerentes entre si e as melhorias propostas são de fácil execução e de grande valia para essa população.
\end{abstract}

Palavras-chave: Lateralidade Funcional, Atividades Cotidianas, Estudantes.

\section{Left-handed graduate students: difficulties found and proposals presented to daily life activities}

\begin{abstract}
This research aimed to identify, through the application of questionnaires, the daily difficulties of left-handed college students and to list their proposals to solve the problems found. One hundred thirty-eight left-handed students participated in the study. They answered a questionnaire composed of eight essay questions and five alternative questions which included information on laterality, school, labor and daily life activities, and on suggestions to improve their activities. It was verified that the main difficulties reported by left-handed college students on daily life, work, and school environment are opening cans, bad arrangement of objects in space, and inadequacy of school desks, respectively. Aiming to improve the performance of their activities within these three aspects, the most cited suggestions are related to improvement/availability of left-handed adapted desks and development of objects adapted to this group. The study shows data on difficulties and coherent suggestions, and the advances proposed are of easy solution and great value for such population.
\end{abstract}

Keywords: Functional Laterality, Activities of Daily Living, Students.

Autor para correspondência: Dernival Bertoncello, Departamento de Fisioterapia Aplicada, Universidade Federal do Triângulo Mineiro, Rua Capitão Domingos, 309, B. Abadia, CEP 38025-010, Uberaba, MG, Brasil, e-mail: bertoncello@fisioterapia.uftm.edu.br 


\section{Introdução}

A evolução tecnológica presenciada nos últimos anos proporcionou grandes benefícios para a humanidade. No entanto, ocorrem vários problemas na interface entre indivíduos e a tecnologia. Dentre eles destacam-se aqueles que envolvem a manipulação de instrumentos e a questão da dominância manual.

A lateralidade é a assimetria de preferência da mão: a grande proporção dos seres humanos prefere utilizar a mão direita na realização de tarefas de mão única (PERELLE; EHRMAN, 1994; PETERS; REIMERS; MANNING, 2006), sendo que essa parece ser uma característica exclusiva de nossa espécie (BYRNE et al., 2006; HOPKINS, 2006). Assim, pode-se considerar a dominância manual como sendo a assimetria lateral humana relacionada ao seu genótipo, caracterizada pelo uso preferencial, parcial ou total de apenas uma mão para realizar tarefas manuais. As classificaçôes são: destros; canhotos ou sinistros; e ambidestros (PASCHOARELLI et al., 2008).

De forma geral e simplificada, indivíduos destros apresentam dominância cerebral à esquerda, já os canhotos, ou sinistros, apresentam dominância cerebral à direita, o que é definido por alguns autores como destralidade ou sinistralidade verdadeira. Já os indivíduos ambidestros são aqueles que não apresentam predomínio claro de dominância cerebral, ocorrendo o uso indiscriminado dos dois lados do corpo (SPRINGER; DEUTSCH, 1998).

Preferência autorreferida da mão tem sido usada como uma medida de lateralidade. McManus (2002) sugeriu que a preferência para a mão escrita poderia ser a medida mais adequada de preferência da mão. $\mathrm{Na}$ verdade, a autoclassificação da lateralidade em geral recai sobre a mão que escreve (PERELLE; EHRMAN, 1994) e a lateralidade classificada com base na escrita está altamente correlacionada com outras medidas de preferência da mão, como jogar uma bola ou segurar uma raquete (ANNETT, 1970, 2009).

De acordo com Raymond et al. (1996), 10\% a $13 \%$ da população têm a preferência no uso da mão esquerda, ou seja, são canhotos. Por ser minoria, essa populaçáo utiliza equipamentos desenvolvidos para destros, produzidos para atender a maioria da população, já que grande parte das ferramentas, utensílios, equipamentos de escritório, eletrodomésticos, roupas, instrumentos médicos, artigos esportivos, armas e instalaçôes públicas é feita para as pessoas que utilizam a mão direita. Assim, muitos canhotos têm de suportar inconveniências ou dificuldades na realização de atividades diárias (JUNG; JUNG, 2009).

Ao lançar um novo produto no mercado, pensa-se em fatores importantes como custo, acessibilidade, estética, praticidade, entre outros. A assimetria, que permitiria um objeto ser utilizado por um destro ou por um canhoto, nem sempre é considerada (MACHADO, 1982). No entanto, as dificuldades encontradas pelos canhotos podem ocorrer desde o manuseio de utensílios do dia a dia, como tesouras, abridores de lata e maçanetas, até situações escolares que exigem procedimentos da esquerda para a direita como, por exemplo, a leitura e a escrita nas línguas ocidentais (MACHADO, 1982). O enfoque ergonômico seria um meio importante para analisar esse problema. A Ergonomia de Produto procura estudar a concepção de uma ferramenta de modo que seu usuário não venha a ter qualquer lesão decorrente de má postura e mau manuseio. Os produtos são ergonomicamente projetados quando possuem características e efeitos desejáveis sobre ou para as pessoas, ou seja, quando a operaçáo deles pode ser feita em posição e postura anatomicamente aceitáveis, entre outros aspectos (RADWIN; HANEY, 1996), o que atualmente não ocorre para a população de canhotos.

Assim, estudos que avaliem a percepçáo das dificuldades encontradas por esses indivíduos poderão auxiliar na identificaçáo dos problemas rotineiros e evidenciar a necessidade de desenvolver e divulgar produtos adaptados para essa população.

Portanto, o objetivo deste trabalho foi identificar as dificuldades cotidianas de universitários canhotos e as propostas levantados por eles para solucioná-las.

\section{Metodologia}

\subsection{Tipo de estudo}

Trata-se de um estudo do tipo descritivo e de natureza quantitativa, desenvolvido mediante a realizaçáo de pesquisa bibliográfica e de campo.

A pesquisa bibliográfica se deu a partir de levantamento efetivado nas bases de dados Medline, Pubmed e Lilacs.

\subsection{Local e sujeitos}

A pesquisa de campo contemplou a abordagem por conveniência de 135 estudantes de diferentes cursos no campus da Universidade de Uberaba que, após afirmação da dominância do membro 
superior esquerdo para escrita, foram convidados a participar como voluntários do presente estudo. $\mathrm{O}$ critério de exclusão foi a falta de disponibilidade do participante para realizar a pesquisa.

O trabalho foi submetido à avaliação do Comitê de Ética em Pesquisa (CEP) em Seres Humanos da Universidade de Uberaba e foi aprovado conforme processo CAAE n. 0018.0.227.000-06. Todos os estudantes voluntários assinaram o Termo de Consentimento Livre e Esclarecido, que continha as informaçóes referentes à pesquisa, conforme determinação do CEP.

\subsection{Procedimentos de coleta de dados}

Os estudantes canhotos responderam a um questionário composto por oito perguntas abertas e cinco perguntas fechadas que englobavam informações sobre a lateralidade, atividades estudantis, laborais, de vida diária e a sugestão para melhorias nas atividades executadas por eles, caso houvesse relato de dificuldade nos itens anteriores. Os estudantes interpretaram e responderam individualmente às questôes.

Algumas das interrogaçóes presentes no questionário eram: Você tem habilidade com as duas mãos? Você encontra ou encontrou alguma dificuldade na atividade estudantil, no trabalho, ou nas atividades de vida diária por ser canhoto? O que você gostaria de sugerir para melhorar suas atividades diárias e/ou o local onde estuda/trabalha?

Cada participante pôde relatar mais de uma dificuldade e/ou sugestáo. Os relatos foram organizados em um banco de dados com auxílio do programa Microsoft Excel ${ }^{\oplus}$ sendo as respostas de todos os estudantes agrupadas pela similaridade e apresentadas em três blocos: Dificuldades encontradas nas atividades diárias; Dificuldades encontradas nas atividades estudantis; e Dificuldades encontradas no trabalho.

Os resultados foram analisados de forma descritiva, a partir da categorização das respostas ao questionário, por similaridade de açóes.

\section{Resultados}

Foram abordados 135 estudantes no campus da universidade. Desses, 62 eram homens e 73, mulheres. A idade deles variou entre 17 e 38 anos $(\mathrm{M}=21,89, \mathrm{DP}= \pm 4,29)$.

Os resultados referentes às dificuldades encontradas pelos canhotos, classificadas nas três, são apresentados na Tabela 1 .

Tabela 1. Dificuldades relatadas pelos canhotos.

\begin{tabular}{lcc}
\hline \multicolumn{1}{c}{ Dificuldades encontradas pelos canhotos } & N & $\%$ \\
\hline Dificuldades encontradas nas atividades diárias & & \\
Abrir latas & 50 & 40,7 \\
Atividades em geral & 30 & 24,4 \\
Utilizar tesoura & 25 & 20,3 \\
Alimentação & 11 & 8,94 \\
Objetos padrão & 6 & 4,85 \\
Falta de orientação nos ensinamentos para os canhotos & 1 & 0,81 \\
Total & 123 & 100 \\
Dificuldades encontradas nas atividades estudantis & & \\
Carteiras escolares & 62 & 77,5 \\
Escrever & 9 & 11,25 \\
Manuseio estudantil geral & 9 & 11,25 \\
Total & 80 & 100 \\
Dificuldades encontradas no trabalho & & \\
Disposição dos objetos no espaço & 21 & 51,22 \\
Força e coordenação & 9 & 21,96 \\
Atividades manuais & 6 & 14,65 \\
Posicionamento adequado para realizar tarefas & 2 & 4,88 \\
Abrir latas & 1 & 2,43 \\
Cadeira sem apoio para os braços & 1 & 2,43 \\
Sensibilidade diminuída na realização de exame físico & 1 & 2,43 \\
Total & 41 & 100 \\
\hline
\end{tabular}


Cinquenta e nove estudantes (43,70\%) relataram não apresentar qualquer tipo de dificuldade nas atividades de vida diária. Os 76 restantes (56,30\%) relataram uma ou mais dificuldades, registrando-se 123 apontamentos.

Atividades diárias consideradas similares foram agrupadas em um mesmo item, de modo a facilitar a visualização dos dados. Dessa forma, no item denominado "atividades em geral" foram englobadas ações como escrever, bordar, passar roupas, fazer crochê e tricô, amarrar cadarço, escovar os dentes, varrer casa, torcer roupas e apertar parafusos. No item "alimentação" constam dificuldades para cortar alimentos, descascar frutas e verduras, utilizar talheres. E do item "objetos padrão" fazem parte as dificuldades encontradas no manuseio de objetos específicos como mouses, cadernos espiral e abrir geladeira.

Em relação às atividades estudantis, 63 (46,66\%) entrevistados relataram não apresentar nenhum tipo de dificuldade. Setenta e dois entrevistados $(53,34 \%)$, uma ou mais dificuldade, sendo que, no total, foram evidenciados 80 apontamentos.

O item "carteiras escolares" abrangeu falta de carteiras específicas para canhotos, falta de mesa com cadeira e má disposição das carteiras. O item "escrever" englobou dificuldades como escrever no quadro-negro, utilizar caderno espiral e borrar as folhas ao escrever. Faz parte do item "manuseio estudantil geral" as dificuldades na realização de técnicas relacionadas à especificidade de cada curso, falta de equipamentos na universidade, manuseio de ferramentas em laboratório e dificuldade em utilizar mouse e tesoura.

Em relação ao ambiente de trabalho, 98 (72,60\%) dos entrevistados relataram não apresentar nenhum tipo de dificuldade. Os 37 restantes $(27,41 \%)$ relataram uma ou mais dificuldade apresentando, no total, 41 apontamentos.

No item Disposição dos objetos no espaço estáo incluídas dificuldades como acomodação de computadores, disposição de objetos sobre a mesa, localização do mouse e do teclado numérico à direita, utilização de materiais que ficam localizados do lado direito. $\mathrm{O}$ item Força e coordenação engloba dificuldades como pegar objetos pesados com a mão direita, paginar processos jurídicos, escrever, fazer projetos de engenharia civil, cumprimentar alguém. Em Atividades manuais constam dificuldades com o manuseio de equipamentos como pipetas de laboratório, porta-agulha, maquinário de marcenaria e equipos de soro.
Ainda, conforme relatos dos estudantes participantes da pesquisa, há algumas sugestôes que podem ser implementadas no cotidiano a fim de amenizar os problemas listados anteriormente (Tabela 2).

\section{Discussão}

De acordo com Noback, Strominger e Demarest (1999), a plasticidade cerebral é a capacidade de modificar sua própria morfologia e o tipo de resposta aos estímulos provenientes do meio interno e/ou externo. O canhoto recebe constantes estímulos do meio externo, pois sempre realiza atividades que exigem adaptaçáo devido à falta de objetos projetados para seu uso. No adulto, a plasticidade cerebral manifesta-se como uma resposta às alteraçóes de níveis hormonais, aprendizado de novas habilidades, resposta a alteraçôes do meio e também lesôes, justificando a relação do canhoto com a adaptação às situaçôes cotidianas. Este estudo, embora não apresente análise específica sobre plasticidade cerebral, corrobora a informação acima, uma vez que há uma grande porcentagem de indivíduos canhotos que não manifestaram qualquer dificuldade nos diversos campos estudados, isto é, apresentam-se adaptados ao meio em que vivem.

Por outro lado, há indivíduos que não se adaptam ou sentem-se desconfortáveis com o fato de a maioria dos equipamentos e ambientes existentes serem produzidos para destros.

Semelhante ao comportamento humano, o curso da lateralidade também pode se alterar devido às influências negativas, com causas, sintomas e consequências possíveis de afetar o seu desenvolvimento. Assim, quando o adulto colabora com a criança ao afirmar sua própria lateralidade, permite-lhe exercer livremente suas experiências motoras. Ao realizar as atividades com sua dominância natural, a criança pode não apresentar futuramente sinais de imperfeição e lentidão motoras, dislexia, problemas de estruturação temporal e espacial, dentre outros (SERAFIN; PERES; CORSEUIL, 2000). Isso propiciaria aos

Tabela 2. Sugestões apresentadas pelos canhotos.

\begin{tabular}{lcc}
\hline \multicolumn{1}{c}{ SUGESTÕES } & N & \% \\
\hline Carteiras para canhotos & 79 & 56,4 \\
Objetos adaptados & 43 & 30,7 \\
Método de ensino & 8 & 5,71 \\
Adaptação de ambientes & 6 & 4,29 \\
Coordenação motora & 4 & 2,86 \\
Total & 140 & 100 \\
\hline
\end{tabular}


indivíduos adultos as dificuldades citadas nos relatos dos itens Alimentação, Manuseio estudantil geral, Força e coordenação e Atividades manuais.

A fim de eliminar as dificuldades citadas acima, os canhotos manifestaram suas sugestôes nos itens Coordenação motora e Método de ensino. Percebe-se certa carência de informaçóes quanto ao manuseio de alguns instrumentos e o desconhecimento no tratamento da pessoa canhota pelos pais e educadores, que muitas vezes forçam a criança a mudar sua condição, tentando que realize as tarefas com a mão direita. Assim, seria importante voltar a atenção para a confecção de material oportunizador de ensino, seja ele de qualquer instância, para auxiliar educadores, pais e o próprio canhoto.

Todos os grupos salientados representam significativamente a importância de voltar à atenção as variadas dificuldades encontradas pelos canhotos cotidianamente. Essas dificuldades representam a falta ou proporção reduzida de produtos para o manuseio diário que atendam a oferta e procura de mercado. Logo, para que a pessoa canhota possa desenvolver suas atividades cotidianas confortavelmente, comparativamente à destra, a criação e adaptação de objetos específicos que reduzam a desavença do uso entre o equipamento e o usuário são necessárias, bem como a aptidão deles para o manuseio com diferentes mãos, direita e esquerda (PASCHOARELLI et al., 2008). Tal afirmativa está comprovada pela manifestaçáo dos entrevistados quando citaram a necessidade dos itens "carteiras para canhotos" e "objetos adaptados" para as atividades cotidianas. Os instrumentos citados são confeccionados, porém sua divulgação e disponibilidade comercial são escassas.

A realização de atividades sem ocasionar maior esforço, insatisfação e impactos na saúde do usuário dependem da boa interaçáo dele com o ambiente, tendo como resultado a sensação de conforto. Quando o espaço físico atende às necessidades dos usuários tanto em termos funcionais (físico-cognitivos) quanto formais (psicológicos), a execução das atividades é agradável (VILLAROUCO; ANDRETO, 2008). Nesse sentido, Paschoarelli e Gil Coury (2000) afirmam que as ferramentas manuais necessitam apresentarem-se aptas para uso com as diferentes máos, direita e esquerda, o que deve contribuir para a melhoria da usabilidade dos produtos, minimizando assim os "atritos" entre o equipamento e o usuário.

Além das atividades de vida diária e estudantis, torna-se indispensável também a adaptação de ambientes de trabalho para atender náo só a demanda de produtividade, mas associar a necessidade de conforto e satisfação laboral de destros e canhotos, destacadas nos itens Posicionamento para realizar as tarefas e Disposição dos objetos no espaço.

Paschoarelli et al. (2008) indicam a necessidade de desenvolvimento de produtos direcionados a canhotos, já que essa faixa da população apresenta particularidades que podem influenciar tanto o desempenho quanto a percepção de uso de um instrumento manual em atividades ocupacionais, prevenindo a ocorrência de distúrbios musculoesqueléticos em atividades que exigem o uso de instrumentos manuais.

Em relação ao citado acima, Al-Johany (2013) realizou um levantamento com estudantes e estagiários de Odontologia canhotos sobre os problemas enfrentados por eles em sua educação ou prática da Odontologia. Mais da metade relataram algum problema. Desses, $84,5 \%$ responderam que a instituição não está devidamente equipada para acomodar os canhotos e $34 \%$ concordaram que o uso das instalações odontológicas por um canhoto pode causar complicações musculoesqueléticas.

Já Makay, Icoz e Ersin (2008) investigaram o papel do canhoto durante a cirurgia endoscópica, encontrando como resultado que $50 \%$ dos cirurgióes canhotos acreditavam que a cirurgia endoscópica precisava ser modificada para o cirurgião endoscópico canhoto, concluindo que ela tem impacto no conforto lateralidade-relacionados e que modificações técnicas devem ser garantidas pata cirurgióes canhotos.

Ainda, Bhushan e Khan (2006) estudaram a associação entre lateralidade e propensão a acidentes em motoristas de locomotivas, encontrando que canhotos eram mais propensos a se envolverem em acidentes e é possível que isso possa estar relacionado com o design das cabines de locomotiva.

Por se tratar de um assunto pouco pesquisado, há necessidade de outros estudos que colaborem com o desenvolvimento de produtos para essa populaçáo, reduzindo o desconforto e evitando possíveis disfunçóes causadas pelas condiçóes atuais e dos ambientes.

\section{Conclusões}

Verificou-se que as maiores dificuldades relatadas por estudantes universitários canhotos na vida cotidiana, no trabalho e no ambiente estudantil, respectivamente, são abrir latas, má disposição dos objetos no espaço e inadequação das carteiras escolares. A fim de melhorar a execução de suas atividades nessas três esferas, as sugestôes mais citadas estâo relacionadas à melhoria/disponibilidade das carteiras para canhotos e ao desenvolvimento de objetos adaptados para esse grupo. 
O presente estudo indica dados sobre dificuldades e sugestóes que são coerentes - as melhorias propostas são de fácil resolução e de grande valia para essa população.

\section{Referências}

AL-JOHANY, S. S. A survey of left-handed dental students and interns in Saudi Arabia. Journal of Dental Education, Washington, v. 77, n. 1, p. 105-112, 2013.

ANNETT, M. A. Classification of hand preference by association analysis. British Journal of Psychology, London, v. 61, n. 3, p. 303-321, 1970. http://dx.doi. org/10.1111/j.2044-8295.1970.tb01248.x

ANNETT, M. A. Patterns of hand preference for pairs of actions and the classification of handedness. British Journal of Psychology, London, v. 100, n. 3, p. 491-500, 2009. PMid:18851767. http://dx.doi. org/10.1348/000712608X357867

BHUSHAN, B.; KHAN, S. M. Laterality and accident proneness: a study of locomotive drivers. Laterality, London, v. 11, n. 5, p. 395-404, 2006.

BYRNE, R. A. et al. Does Octopus vulgaris have preferred arms? Journal of Comparative Psychology, Washington, v. 120, n. 3, p. 198-204, 2006. PMid:16893257. http:// dx.doi.org/10.1037/0735-7036.120.3.198

HOPKINS, W. D. Comparative and familial analysis of handedness in great apes. Psychological Bulletin, Washington, v. 132, n. 4, p. 538-559, 2006. PMid:16822166 PMCid:PMC2063575. http://dx.doi. org/10.1037/0033-2909.132.4.538

JUNG, H. S.; JUNG, H. S. Hand dominance and hand use behaviour reported in a survey of 2437 Koreans. Ergonomics, London, v. 52, n. 11, p. 1362-1371, 2009. PMid:19851904. http://dx.doi.org/10.1080/00140130903067805

MACHADO, D. V. M. Meu filho é canhoto: o que saber e como agir em relação ao canhotismo. São Paulo: ALMED, 1982.

MAKAY, O.; ICOZ, G.; ERSIN, S. Surgeon's view on the limitations of left-handedness during endoscopic surgery. Journal of Laparoendoscopic and Advanced Surgical Techniques: Part A, Larchmont, v. 18, n. 2, p. 217-221, 2008. PMid:18373447. http://dx.doi.org/10.1089/ lap. 2007.0091
MCMANUS, I. C. Right hand, left hand: the origins of asymmetry in brains, bodies, atoms and cultures. Cambridge: Harvard University Press, 2002.

NOBACK, C. R.; STROMINGER, N. L.; DEMAREST, R. J. Neuroanatomia: estrutura e função do sistema nervoso humano. 5. ed. Tradução Ivone Castilho Benedetti. São Paulo: Premier, 1999.

PASCHOARELLI, L. C. et al. Manipulação de instrumentos manuais e a influência da dominância nos aspectos perceptivos e de desempenho: uma contribuiçáo do design ergonômico. Revista Assentamentos Humanos, Marília, v. 8, n. 1, p. 9-23, 2008.

PASCHOARELLI, L. C.; GIL COURY, H. J. C. Aspectos ergonômicos e de usabilidade no design de pegas e empunhaduras. Estudos em Design, Rio de Janeiro, v. 8, n. 1, p. 79-101, 2000.

PERELLE, I. B.; EHRMAN, L. An international study of human handedness: the data. Behavior Genetics, New York, v. 24, n. 3, p. 217-227, 1994. http://dx.doi. org/10.1007/BF01067189

PETERS, M.; REIMERS, S.; MANNING, J. T. Hand preference for writing and associations with selected demographic and behavioral variables in 255,100 subjects: the BBC internet study. Brain and Cognition, New York, v. 62 , n. 2, p. 177-189, 2006. PMid:16797814. http:// dx.doi.org/10.1016/j.bandc.2006.04.005

RADWIN, R. G.; HANEY, J. T. An ergonomics guide to hand tools. Virginia: American Industrial Hygiene Association, 1996.

RAYMOND, M. et al. Frequency-dependent maintenance of left handedness in humans. Proceedings: Biological Sciences, Londres, v. 263, n. 1377, p. 1627-1633, 1996.

SERAFIN, G.; PERES, L. S.; CORSEUIL, H. X. Lateralidade: conhecimentos básicos e fatores de dominância em escolares de 7 a 10 anos. Caderno de Educação Física, Marechal Candido Rondon, v. 2, n. 1, p. 11-30, 2000 .

SPRINGER, S. P.; DEUTSCH, G. Cérebro direito, cérebro esquerdo. Tradução de Thomaz Yoshiura. São Paulo: Summus, 1998.

VILLAROUCO, V.; ANDRETO, L. F. M. Avaliando desempenho de espaços de trabalho sob o enfoque da ergonomia do ambiente construído. Production, São Paulo, v. 18, n. 3, p. 523-539, 2008.

\section{Contribuição dos Autores}

Elisângela de Assis Amaro realizou a coleta, organização de fontes, análise e redação do texto. Karoline Cipriano Raimundo e Núbia Tomain Otoni realizaram a organização de fontes, análise e redação do texto. Patrícia Beatriz Silva Bonfim participou da coleta dos dados. Isabel Aparecida Porcatti de Walsh participou da análise e revisão do texto. Dernival Bertoncello foi responsável pela orientação e revisão do texto. 